\title{
A Case of Recurrent Fever with Chest and Abdominal Pain -A Rare Presentation of Autoinflammatory Disease
}

\author{
Shuixiang Deng $\mathrm{MD}^{1 \# *}$, Xiaoyan Yu MD ${ }^{1 \#}$, Jun Shen $\mathrm{AD}^{1}$, Umut Ocak $\mathrm{AD}^{2}$ and Ye Gong $\mathrm{AD}^{1}$ \\ ${ }^{1}$ Department of Critical Care Medicine, Huashan Hospital affiliated to Fudan University, China \\ ${ }^{2}$ Department of Emergency Medicine, University of Health Sciences, Bursa Yuksek Ihtisas Training and Research Hospital, Turkey \\ "Shuixiang Deng and Xiaoyan Yu are the co-first authors of this study, because their contributions to this study are equal.
}

*Corresponding author: Shuixiang Deng, Department of Critical Care Medicine, Huashan Hospital affiliated to Fudan University, Shanghai, No.12 middle urumqi road, Shanghai, 200040, China.

To Cite This Article: Shuixiang Deng MD, Xiaoyan Yu MD, Jun Shen AD, Umut Ocak AD, Ye Gong AD. A Case of Recurrent Fever with Chest and Abdominal Pain -A Rare Presentation of Autoinflammatory Disease. 2020 - 8(6). AJBSR.MS.ID.001340. DOI: 10.34297/AJBSR.2020.08.001340.

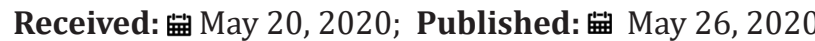

\begin{abstract}
Background: Monogenic autoinflammatory diseases (AID) which are characterized by genetic protein abnormalities involved in innate immunity.

Case presentation: A 28-year-old male patient who presented to our clinic with recurrent fever along with chest pain and abdominal pain. His electrocardiogram test was normal. Due to incomplete intestinal obstruction, initially, laparotomy was considered for the patient. Based on the family history with repeated fever and abdominal pain in two of his second degree relatives, we timely carried out second-generation gene sequencing as the diagnosis of TNF receptor-associated periodic syndrome (TRAPS) was highly suspicious. Genetic testing was positive and heterozygous for the TRAPS sequence mutation (the mutant gene was TNFRSF1A), hence, we successfully avoided surgical intervention. Here, we discussed the diagnosis process and current understanding of the TRAPS treatment
\end{abstract}

Conclusions: In clinical practice, physicians should keep genetic diseases in mind and carry out early gene sequencing for definitive diagnosis in patients with repeated fever and with no obvious evidence of infection. A high index of suspicion for early detection and prompt treatment would prevent disease progression, save medical resources and reduce costs.

Keywords: Recurrent fever; Abdominal pain; Autoinflammatory disease; TNF receptor-associated periodic syndrome; TNFRSF1A

Abbreviations: AID: Monogenic Autoinflammatory Diseases; TRAPS: TNF Receptor-Associated Periodic Syndrome; FMF: Familial Mediterranean Fever; MKD: Mevalonate Mina Deficiency; CAPS: Cryopyrin-Associated Periodic Syndrome; NGS: Next-Generation Sequencing; SHARE: European Project Single Hub and Access Point For Paediatric Rheumatology in Europe.

\section{Background}

Monogenic autoinflammatory diseases (AID) which are characterized by genetic protein abnormalities involved in innate immunity [1] were first recognized nearly 20 years ago as distinct hereditable disorders. Since then, many advances in genomic techniques had led the discovery of new monogenic AID year by year. Main monogenic auto-inflammatory syndromes include Familial Mediterranean Fever (FMF), Mevalonate Mina Deficiency (MKD), Blau Syndrome, TNF receptor-associated periodic syndrome (TRAPS), cryopyrin-associated periodic syndrome (CAPS), and pyogenic arthritis with pyoderma gangrenosum and acne (PAPA)
[2]. They are characterized by elevated inflammatory blood biomarkers during the crisis usually without the presence of an infectious status and without the presence of autoantibodies. Here, we described a case of monogenic AID treated at our institution with good results.

\section{Case Presentation}

A 28-year-old Chinese man presented to the emergency department of a local facility with the complaints of fever, chest pain, and right lower abdominal pain which started almost 24 
hours ago. His past medical history was unremarkable. The patient was married and did not take any medications. He had no history of smoking and /or alcohol used. The blood test obtained at the outside facility for a possible infectious condition revealed C-reactive protein (CRP) as $67.5 \mathrm{mg} / \mathrm{L}$, erythrocyte sedimentation rate (ESR) as $71 \mathrm{~mm} / \mathrm{h}$, and white blood cell (WBC) as $12.08 \times 109 / \mathrm{L}$. He was treated with ceftadine (iv $2.0 \mathrm{q} 8 \mathrm{~h}$ ) and levofloxacin (iv 0.5 qd ) for 5 days, which failed to control the fever. Then, for further evaluation, he was transferred to our hospital. Indeed, the patient had suffered from recurrent fever and abdominal pain since the age of 13 . The fever characteristically occurred 1 or 2 times per year, usually in spring, and the body temperature fluctuated between 36.0 to $40.2{ }^{\circ} \mathrm{C}$. Each febrile episode lasted between 7 to 15 days. His symptoms were triggered by fatigue and irregular diet. He had the diagnosis of appendicitis and underwent surgical resection at the age of 13 when the first episode happened.

On physical examination, his vital signs were within normal limits (blood pressure 98/50 $\mathrm{mmHg}$, pulse rate $80 \mathrm{bpm}$, respiratory rate 15 breaths/min and body temperature 38.6oc). Chest and cardiovascular system's examinations were unremarkable. The abdomen was soft and non-tender but there was a rebound pain in the right lower abdomen. Intestinal rumbling was $3 /$ minute.

Radiologic evaluation of the lungs with computed tomography (CT) and abdominal ultrasound with Doppler revealed no pathological findings. His initial abdominal radiograph upon admission demonstrated incomplete ileus (Figure 1). Further evaluation with lower abdomen CT revealed that left jejunum was disordered and fat gap around the right ileocecal area was slightly blurred, with pelvic fluid (Figure 2). His colonoscopy showed the normal image (Figure 3). The workup for infectious disease including blood cultures as well as workup for human immunodeficiency virus, cytomegalovirus, tuberculosis spot and Epstein-Barr virus infection was negative. Laboratory results showed elevated serum CRP $148 \mathrm{mg} / \mathrm{L}$, ESR $76 \mathrm{~mm} / \mathrm{h}$, procalcitonin $0.25 \mathrm{ng} / \mathrm{ml}$, and WBC $15.0 \times 109 / \mathrm{L}$. All markers of autoimmune disease such as ENA, dsDNA, c-ANCA, p-ANCA, SS-A, SSB, ESR, and rheumatoid factor were negative. Liver, kidney, and cardiac function as well as electrolyte tests were normal.

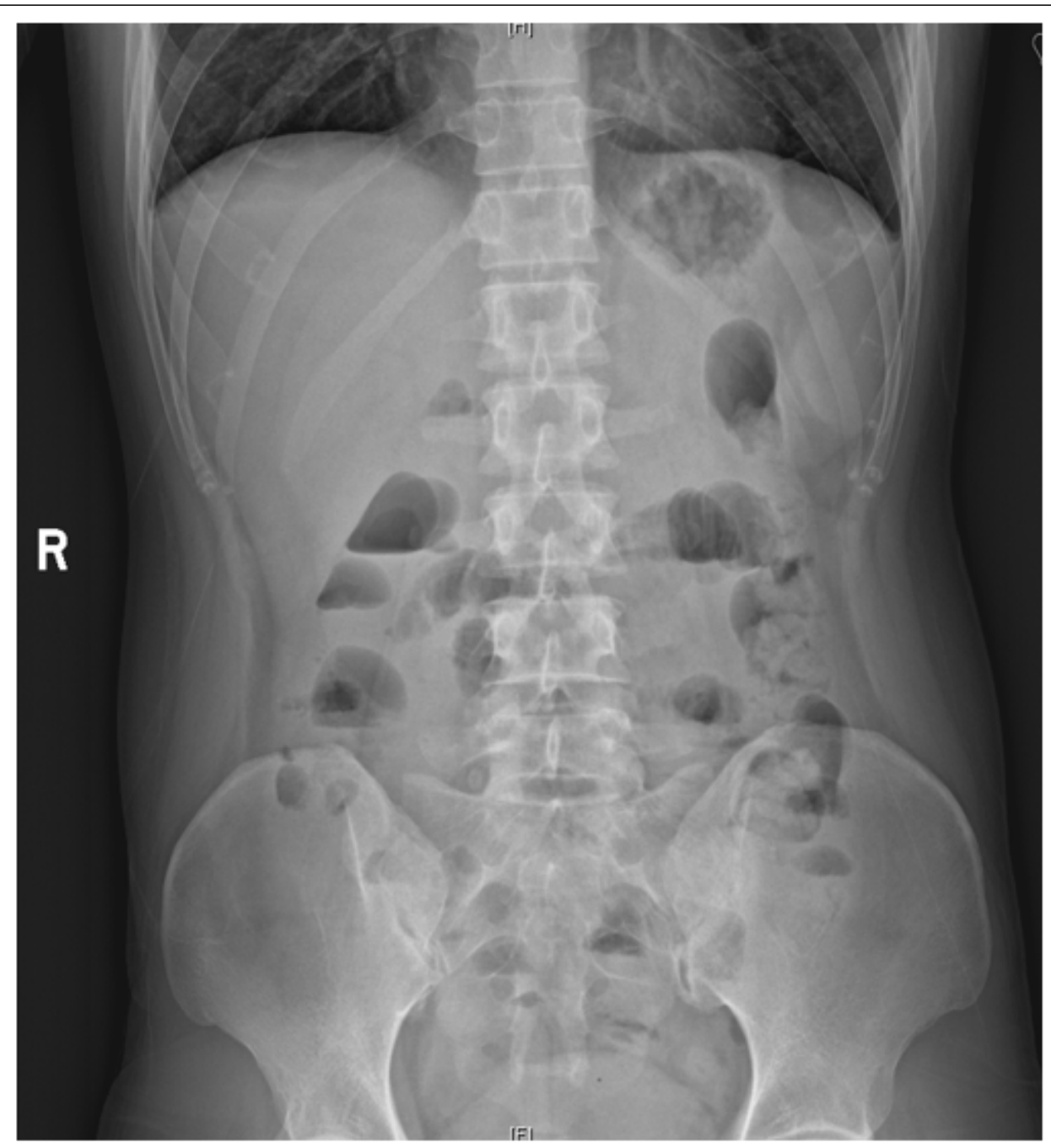

Figure 1: Initial abdominal radiograph of a 28-year-old male patient who presented with the complaints of fever and right lower quadrant abdominal pain showing incomplete ileus. 

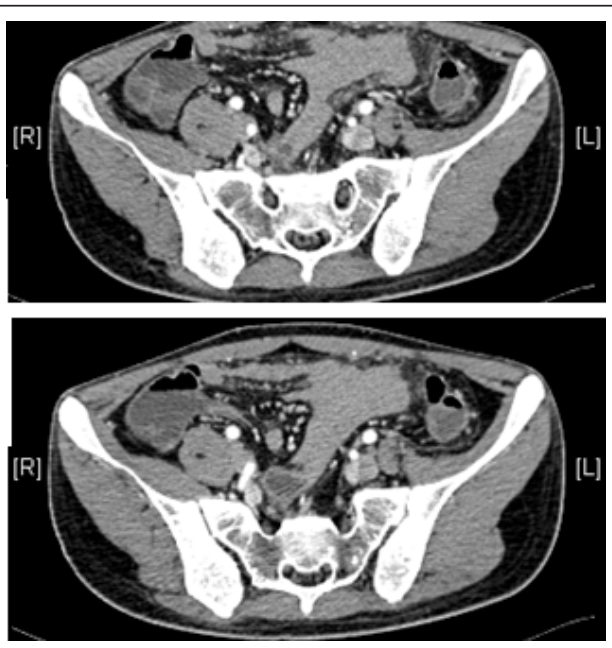

Figure 2: Lower abdomen CT revealed that left jejunum was disordered.

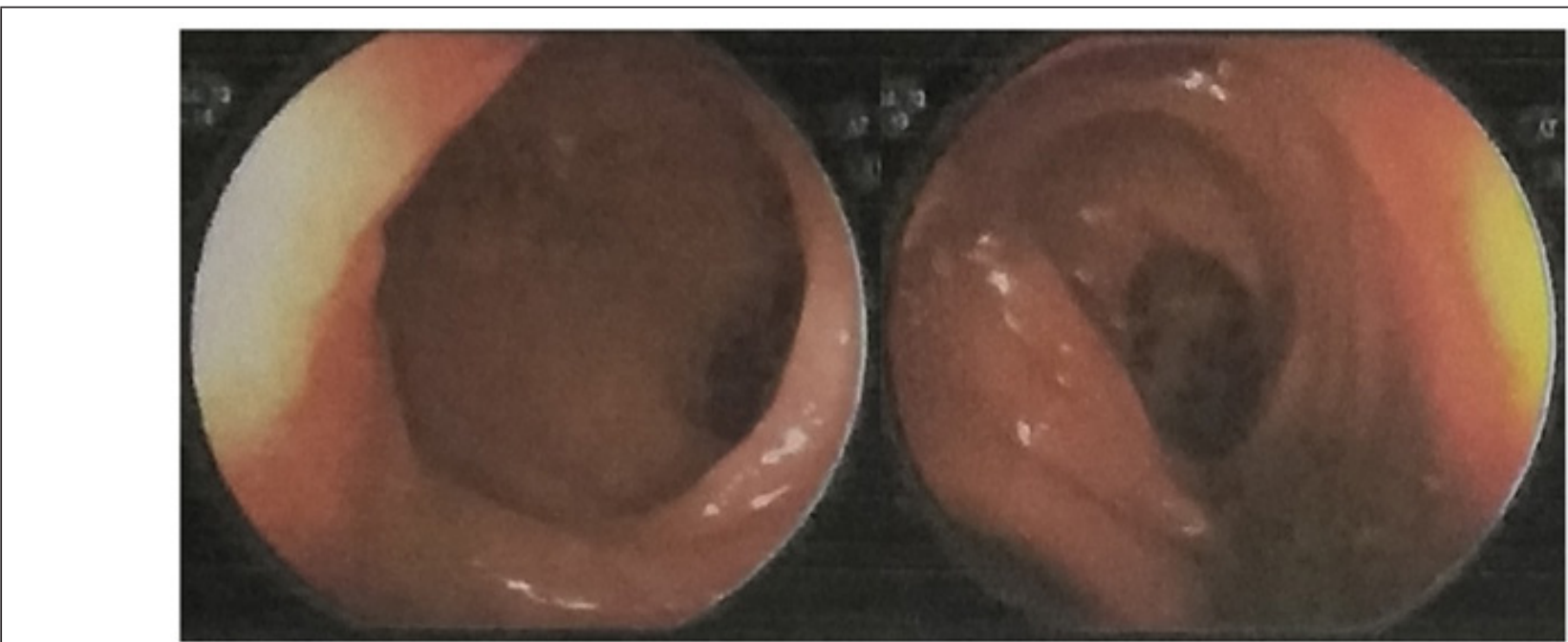

Figure 3: Colonoscopy showed the normal image.

The surgeon prepared an exploratory laparotomy after evaluation, but the patient and his family refused but continue treatment at the hospital. Due to the recurrent febrile episode, the patient was administered Phosphamycin sodium 6g Q8H iv for a week and imipenem cilastatin sodium 2 bottles Q8H iv for ten days but with no benefits. For further diagnosis, colonoscopy was also done and no positive result was reported (Figure 3). His aunty and cousin had similar symptoms, manifesting as repeated fever and abdominal pain for many years. So we highly suspected single-gene AID and sent five bloods (the family of patient, his aunty and cousin) to do the second-generation gene sequencing. Genetic testing was positive and heterozygous for the TRAPS sequence mutation (the mutant gene was TNFRSF1A).

Based on these results, a definitive diagnosis of TRAPS was made, and the patient's further management focused on treating this particular condition with nonsteroidal anti-inflammatory drugs (NSAIDs) for the fever and pain. After 3-month follow-up, he recovered and no fever or abdominal pain occurred.

\section{Discussion and Conclusion}

This case report described a rare and a unique presentation of TRAPS. TRAPS, initially called "familial Hibernian fever" [3], was first described in 1982 in a family of Irish and Scottish descent. TRAPS is a very rare genetic disorder with autosomal dominant transmission and probably represents a large proportion of the patients with fever from all origins, who display inflammatory abdominal pain lasting more than one week [4]. Mean age at onset is 10 years old. Each attack lasts between a few days and a few weeks. Clinical presentation includes fever, abdominal pain, periorbital edema, joint pains and migrating erythema. In addition to a fever, the attacks are characterized in three-fourths of cases by abdominal pain, which may suggest a surgical emergency. When patients present with periodic and recurrent fevers, we must consider autoinflammatory diseases, especially Familial Mediterranean fever, Methylhydroxy valerate kinase deficiency, Cryopyrinopathies, and TNF receptor-associated periodic syndromes. 
The gene that encodes for the TNF type $1 \mathrm{~A}$ receptor is responsible for TRAPS syndrome. Most mutations are related to cysteine in the extracellular partial spatial structure of TNFRSFA1, which reinforces the suspicion of its pathogenicity [5]. The pathogenesis of the disease is not fully understood. The diagnosis was based on clinical data, personal medical history, family history, age of onset (symptom onset), and the duration of onset, which must be determined by genomic DNA analysis of TNFRSF1A mutation [6]. To date, about 50 mutations have been identified in nearly 200 patients who had clinical manifestations consistent with TRAPS [7]. Given to extreme rarity of this disease and the lack of precise clinical classification criteria and/or incomplete gene screening, the diagnosis is often delayed. Therefore, to improve the molecular diagnosis, the next-generation sequencing (NGS) has also been used [8]. In this case, the patient was proved TRAPS by NGS.

Previous studies have showed that Colchicine was not effective in TRAPS. Pulses of glucocorticoids were partially effective in TRAPS but high doses were required [7]. A recent trial has shown that canakinumab was safe and efficient for reducing inflammatory attacks in patients with TRAPS [9]. Recently SHARE (European project single hub and access point for paediatric rheumatology in Europe) has developed recommendations for the management and treatment of AID [10].

The treatment suggestions for TRAPS are as follows:

A. NSAIDs may provide symptom relief during inflammatory attacks;

B. Short-term glucocorticoids, with or without NSAIDs, are effective in stopping inflammatory attacks;

C. The beneficial effect of corticosteroids can decline over time;

D. IL-1 blockade is beneficial in the majority of patients with TRAPS;

E. Etanercept can be effective in some patients, but the effect might decline over time;

F. With frequent attacks and/or subclinical inflammation between attacks, maintenance therapy with IL-1 blockade or etanercept is recommended to limit steroids;

G. If one IL-1 blocking agent at adequate dose is ineffective or intolerable, a switch to etanercept or another IL-1 blocking agent should be considered. Similarly, if etanercept is ineffective, a switch to an IL-1 blocking agent should be considered;

H. Although a beneficial effect is reported in a few cases, the use of anti-TNF monoclonal antibodies is not advised, due to the possible detrimental effect.

TRAPS is a potentially devastating disease process. Therefore, we should pay close attention to those patients with recurrent fever with chest and abdominal pain. Similar symptoms in family members could provide a clue for the diagnosis. Otherwise, the disease can easily be misdiagnosed as an acute abdomen and treated with surgery. In clinical practice, doctors should consider genetic diseases and carry out early gene sequencing for definite diagnosis in patients with repeated fever without obvious evidence of infection. In general, the treatment of these very rare diseases must be discussed in a multidisciplinary consultation meeting with experts who are used to treat these patients. A high index of suspicion for early detection and prompt treatment can prevent disease progression and save medical resources and costs.

\section{Declarations}

A. Ethics approval and consent to participate: We have obtained the written consent of patient to participate in the genetic testing.

B. Consent for publication: We also have obtained the written consent of patient for publication of the patient's family's clinical details and clinical images was obtained from the patient's family.

C. Availability of data and material: The datasets used and/ or analysed during the current study are available from the corresponding author on reasonable request.

D. Competing interests: None

E. Funding: The grants No. are18140901100, 2016ZB020201 and 20184Y0064 and the funder (Shuixiang Deng) are responsible for collecting patient data and writing articles.

F. Authors' contributions: SX Deng was responsible for collecting data and writing articles; XY Yu was responsible for providing medical history and collecting data; J Shen was responsible for patient management and article guidance; U Ocak was responsible for revising articles, and Y Gong was responsible for article guidance.

\section{References}

1. Georgin-Lavialle S, Fayand A, Rodrigues F, Bachmeyer C, Savey L, Grateau G (2019) Autoinflammatory diseases: State of the art. Presse medicale 48: e25-e48.

2. Georgin-Lavialle S, Rodrigues F, Hentgen V, Fayand A, Quartier P, et al. (2018) Clinical overview of auto-inflammatory diseases. La Revue de medecine interne 39(4): 214-232.

3. Williamson LM, Hull D, Mehta R, Reeves WG, Robinson BH, et al. (1982) Familial Hibernian fever. The Quarterly journal of medicine 51(204): 469-480.

4. Georgin-Lavialle S, Kone-Paut I, Delaleu J, Sarrabay G, Grateau G, et al. (2018) Tumor necrosis receptor associated periodic syndrome (TRAPS): State of the art. La Revue de medecine interne 39(4): 256-264.

5. Lachmann HJ, Papa R, Gerhold K, Obici L, Touitou I, et al. (2014) The phenotype of TNF receptor-associated autoinflammatory syndrome (TRAPS) at presentation: a series of 158 cases from the Eurofever/ EUROTRAPS international registry. Annals of the rheumatic diseases 73(12): 2160-2167. 
6. Federici S, Sormani MP, Ozen S, Lachmann HJ, Amaryan G, et al. (2015) Evidence-based provisional clinical classification criteria for autoinflammatory periodic fevers. Annals of the rheumatic diseases 74(5): 799-805.

7. Sag E, Bilginer Y, Ozen S (2017) Autoinflammatory Diseases with Periodic Fevers. Curr Rheumatol Rep 19(7): 41.

8. Rusmini M, Federici S, Caroli F, Grossi A, Baldi M, et al. (2016) Nextgeneration sequencing and its initial applications for molecular diagnosis of systemic auto-inflammatory diseases. Annals of the rheumatic diseases 75: 1550-1557.

9. De Benedetti F, Gattorno M, Anton J, Ben-Chetrit E, Frenkel J, et al. (2018) Canakinumab for the Treatment of Autoinflammatory Recurrent Fever Syndromes. The New England journal of medicine 378(20): 1908-1919.

10. Ter Haar NM, Oswald M, Jeyaratnam J, Anton J, Barron KS, et al. (2015) Recommendations for the management of autoinflammatory diseases. Annals of the rheumatic diseases 74(9): 1636-1644. 